\section{OP0031 SHARED AND DISTINCT GUT MICROBIOME SIGNATURES IN PATIENTS WITH AXIAL SPONDYLOARTHRITIS AND ITS RELATED IMMUNE- MEDIATED DISEASES}

V. Rios Rodriguez ${ }^{1}$, M. Essex ${ }^{2,3}$, J. Rademacher ${ }^{1,4}$, F. Proft ${ }^{1}$, U. Löber, ${ }^{2,3}$ L. Marko $^{3,5}$, U. Pleyer $^{6}$, B. Siegmund ${ }^{1}$, D. Poddubnyy ${ }^{1,7}$, S. Forslund $d^{2,3,5,8}$ ${ }^{1}$ Charité - Universitätsmedizin Berlin, Campus Benjamin Franklin, Gastroenterology, Infectiology and Rheumatology, Berlin, Germany; ${ }^{2} \mathrm{Helmholtz}$ Association of German Research Centers e.V., Max Delbrück Center for Molecular Medicine (MDC), Berlin, Germany; ${ }^{3}$ Experimental and Clinical Research Center, a Joint Cooperation Between the Charité Medical Faculty and the Max Delbrück Center for Molecular Medicine, Experimental and Clinical Research Center, Berlin, Germany; ${ }^{4}$ Berlin Institute of Health, Clinical Research Unit, Berlin, Germany; ${ }^{5}$ German Center for Cardiovascular Research (DZHK), Partner Site Berlin, Berlin, Germany; ${ }^{6}$ Charité Campus Virchow Clinic, Oftalmology, Berlin, Germany; ${ }^{7}$ Deutsches Rheuma-Forschungszentrum Berlin (DRFZ), ein Institut der Leibniz-Gemeinschaft, Epidemiology, Berlin, Germany; ${ }^{8}$ EMBL Heidelberg, Structural and Computational Biology Unit, Heidelberg, Germany

Background: Immune-mediated diseases such as spondyloarthritis (SpA) consistently coincide with dysbiosis of the gut microbiota and frequently present with additional inflammatory pathologies such as Crohn's disease (CD) and acute anterior uveitis (AAU). Deep profiling of gut microbiota may reveal new pathways of how SpA and its related diseases are initiated and perpetuated.

Objectives: To identify the presence of shared and specific gut microbiota signatures for SpA and its related diseases as a whole, as well as for the individual diseases, relative to healthy controls.

Methods: Patients were recruited with a definite diagnosis of axial SpA, AAU or $\mathrm{CD}$ and were compared to controls (patients with back pain and previously ruled out $\mathrm{SpA} / \mathrm{CD} / \mathrm{AAU}$ diagnosis). All patients were naïve to or did not receive treatment with biological disease-modifying antirheumatic drugs for at least 3 months before enrollment of the study. Fecal samples were collected and microbiota composition was determined by 16S rRNA gene sequencing, followed by computational analysis referencing the SILVA138 database. Nonparametric Wilcoxon tests were used to calculate differential abundances between binary groups, and the Spearman correlation was used with continuous covariates. Nested linear models and likelihood ratio tests were used to assess confounding with respect to patient characteristics, HLA-B27 expression, inflammatory markers, and the presence of other immune-mediated diseases.

Results: A total of 300 patients were recruited for the study: 111 axial SpA, $110 \mathrm{AAU}$, and $79 \mathrm{CD}$ patients and were compared to 63 control individuals. Fifty-three of patients were males with an age $($ mean $\pm S D)$ of $39.1 \pm 12.3$ years. The prevalence of HLA-B27 was $63.0 \%$ by patients compared to $7.9 \%$ by control individuals. A multivariate PERMANOVA test between the groups was significant $(p<0.001)$, revealing a difference in overall composition between the groups.

At the phylum level, patients with axial SpA, AAU and CD contained higher abundances of Proteobacteria, Bacteroidetes and Fusobacteria, and lower abundances of Firmicutes and Actinobacteria compared to the control group. At the genus level, patients (with axial SpA, AAU and $\mathrm{CD}$ ) displayed a shared gut microbiome signature differing from that of control individuals. Patients samples were strongly depleted in Blautia compared to the control group. Many of the differentially abundant taxa also correlated with increased inflammation as measured by C-reactive protein (CRP), including a depletion of Fusicatenibacter, Lachnospiraceae FCSO2O and Roseburia, as well as an enrichment of Lactobacillus and Veillonella. By looking at each separate disease phenotype, $C D$ patients differed significantly from the control individuals with respect to many genera. These primarily consisted of depletions in Clostridiales (Roseburia, Coprococcus, Ruminococcaceae), and enrichments of pathogen-harboring genera such as Escherichia-Shigella and Fusobacterium. Axial SpA patients were uniquely enriched in Collinsella and Holdemanella and depleted in Cupriavidus; the enrichment of Lactobacillus and depletion of Blautia observed in all patient groups was also associated to the presence of axial SpA, though confounded by CRP. There were strong taxa associations to the presence of HLA-B27, including enrichment of Asteroleplasma, Coprococcus, Faecalibacterium, Rominococcaceae, Lachnospiraceae NK4A136 and Rikenellaceae.

Conclusion: There is a robust shared taxonomic signature among related immune-mediated diseases, in addition to individual disease phenotype signatures. Patients frequently exhibited a strong depletion in Blautia and an enrichment in Lactobacillus as well as pathogen-harboring genera such as Escherichia-Shigella and Fusobacterium.



Figure 1. Taxa associations within and between the groups resulting from comparing each with the control group and accounting for disease concomitance and patient characteristics $($ FDR $\leq 0.05)$. AAU, anterior acute uveitis; CD, Crohn's disease; SpA, spondyloarthritis.

Disclosure of Interests: None declared

DOI: 10.1136/annrheumdis-2021-eular.1965

\section{OP0032 \\ SINGLE CELL ANALYSIS OF SPONDYLOARTHRITIS REGULATORY T CELLS IDENTIFIES DISTINCT SYNOVIAL GENE EXPRESSION PATTERNS AND CLONAL FATES}

D. Simone ${ }^{1,2}$, F. Penkava ${ }^{1}$, A. Ridley ${ }^{1}$, S. Sansom ${ }^{3}$, H. Al Mossawi ${ }^{1}$, P. Bowness' ${ }^{1}{ }^{1}$ University of Oxford, NDORMS, Oxford, United Kingdom; ${ }^{2}$ Università della Campania “Luigi Vanvitelli,"Dipartimento Medicina di Precisione, Naples, Italy; ${ }^{3}$ University of Oxford, Kennedy Institute of Rheumatology, Oxford, United Kingdom

Background: Regulatory $\mathrm{T}$ cells (Tregs) play an important role in controlling inflammation and limiting autoimmunity, but their phenotypes at inflammatory sites in human disease are poorly understood. Whilst the phenotype and transcriptional profile of Tregs have been studied in some immune mediated conditions, they have been little studied (especially at the single cell level) in synovial fluid in the course of inflammatory arthritis. In Spondyloarthritis (SpA), in particular, where pathogenesis and inflammation is driven by dysregulated effector immunity, the role of the regulatory arm of immunity is largely unknown.

Objectives: We aimed to draw an atlas of Tregs in the context of SpA joint inflammation using single cell RNA sequencing of blood and SF Tregs of patients with Ankylosing Spondylitis (AS) and Psoriatic Arthritis (PsA). Functionally distinct specialised Treg subtypes, and specific changes in transcriptional profile occurring in synovial fluid Tregs, providing an insight on Treg adaptation during inflammation. Furthermore, by coupling gene expression analysis with TCR sequencing, we aimed to describe clonally expanded and likely antigen-driven Tregs in the SF.

Methods: Fluorescent activated cell sorting (FACS) was used to isolate 13,400 memory CD3+ CD45RA-ve CD25+127low Tregs from the blood and synovia fluid (SF) of 2 patients with HLA-B27+ AS presenting with active knee arthritis. Single-cell RNA sequencing (scRNA-seq) using 5' V(D)J 10x Genomics technology allowed both transcriptional definition of Tregs, and exploration of their immune TCR repertoire. Findings were compared to $>3,000 \mathrm{SF}$ and blood Tregs from 3 patients with olygoarticular PsA ${ }^{1}$. Multicolor flow cytometry and in vitro cell-based assays using patient-derived cells were used to confirm and expand at protein and functional level, the findings that emerged from the gene expression analysis.

Results: We report a large scRNAseq dataset (approx. 17,000 cells) comparing Tregs from SpA blood and joints. We identify multiple Treg clusters with distinct transcriptomic profiles, including, among others, a regulatory CD8+ subse expressing cytotoxic markers/genes, and a Th17-like RORC+ Treg subset characterized by IL-10 and LAG-3 expression. Synovial Tregs show upregulation of interferon signature and TNF receptor superfamily genes, and marked clonal expansion, consistent with tissue adaptation and antigen contact respectively. Individual synovial Treg clones map to different clusters indicating cell fate divergence. Finally, we demonstrate that LAG-3 directly inhibits IL-12/23 and TNF 
secretion by patient-derived monocytes, a mechanism with translational potential in SpA.

Conclusion: Our detailed characterization of Tregs at an important inflammatory site illustrates the marked specialization of Treg subpopulations and identifies a broad transcriptional profile upregulated across all synovial regulatory cells. Our TCR analysis provides evidence of Treg clonal expansion, which may be driven by antigen, and confirms functional specialisation of individual clones. We also propose a new insight into a Treg functional mechanism through LAG-3 that suggests a novel therapeutic approach to immune-driven diseases.

REFERENCES:

[1] Penkava et al., Nature Communications, 2020

Disclosure of Interests: Davide Simone: None declared, Frank Penkava: None declared, Anna Ridley: None declared, Stephen Sansom: None declared, Hussein Al Mossawi Employee of: UCB, Paul Bowness Grant/research support from: Regeneron, Celgene/BMS and GSK

DOI: 10.1136/annrheumdis-2021-eular.4278

\section{OP0033 REGULATORY T CELL CD39 EXPRESSION AS A PREDICTOR OF EARLY REMISSION-INDUCTION WITH METHOTREXATE IN NEW-ONSET RHEUMATOID ARTHRITIS}

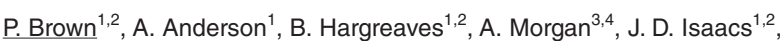
A. Pratt ${ }^{1,2} .{ }^{1}$ Translational and Clinical Research Institute, Faculty of Medical Sciences, Newcastle University, Newcastle upon Tyne, United Kingdom; ${ }^{2}$ Musculoskeletal Unit, Newcastle upon Tyne Hospitals NHS Foundation Trust, Newcastle upon Tyne, United Kingdom; ${ }^{3}$ Leeds Institute of Cardiovascular and Metabolic Medicine, Discovery and Translational Science Department, School of Medicine, University of Leeds, Leeds, United Kingdom; ${ }^{4}$ National Institute for Health Research, Leeds Biomedical Research Centre, Leeds, United Kingdom

Background: The long term outcomes for patients with rheumatoid arthritis (RA) depend on early and effective disease control. Methotrexate remains the key first line disease modifying therapy for the majority of patients, with $40 \%$ achieving an ACR50 on monotherapy(1). There are at present no effective biomarkers to predict treatment response, preventing effective personalisation of therapy. A putative mechanism of action of methotrexate, the potentiation of anti-inflammatory adenosine signalling, may inform biomarker discovery. By antagonism of the ATIC enzyme in the purine synthesis pathway, methotrexate has been proposed to increase the release of adenosine moieties from cells, which exert an anti-inflammatory effect through interaction with ADORA2 receptors(2). Lower expression of CD39 (a cell surface 5-'ectonucleotidase required for the first step in the conversion of ATP to adenosine) on circulating regulatory T-Lymphocytes (Tregs) was previously identified in patients already established on methotrexate who were not responding (DAS28 $>4.0$ vs <3.0)(3). We therefore hypothesised that pre-treatment CD39 expression on these cells may have clinical utility as a predictor of early methotrexate efficacy.

Objectives: To characterise CD39 expression in peripheral blood mononuclear cells in RA patients naïve to disease modifying therapy commencing methotrexate, and relate this expression to 4 variable DAS28CRP remission $(<2.6)$ at 6 months.

Methods: 68 treatment naïve early RA patients starting methotrexate were recruited from the Newcastle Early Arthritis Clinic and followed up for 6 months Serial blood samples were taken before and during methotrexate therapy with peripheral blood mononuclear cells isolated by density centrifugation. Expression of CD39 by major immune subsets (CD4+ and CD8+ T-cells, B-lymphocytes, natural killer cells and monocytes) was determined by flow cytometry. The statistica analysis used was binomial logistic regression with baseline DAS28CRP used as a covariate due to the significant association of baseline disease activity with treatment response.

Results: Higher pre-treatment CD39 expression was observed in circulating CD4+ T-cells of patients who subsequently achieved clinical remission at 6 months versus those who did not (median fluorescence 4854.0 vs 3324.2; $p=$ 0.0108 ; Figure 1-A). This CD39 expression pattern was primarily accounted for by the CD4+CD25 high sub-population (median fluorescence 9804.7 vs $6455.5 ; \mathrm{p}=$ 0.0065 ; Figure 1-B). These CD25 high cells were observed to have higher FoxP3 and lower CD127 expression than their CD39 negative counterparts, indicating a Treg phenotype. No significant associations were observed with any other circulating subset. A ROC curve demonstrates the discriminative utility of differential CD39 expression in the CD4+CD25 high population for the prediction of DAS28CRP remission in this cohort, showing greater specificity than sensitivity for remission prediction(AUC: 0.725; 95\% Cl: 0.53 - 0.92; Figure 1-C). Longitudinally, no significant induction or suppression of the CD39 marker was observed amongst patients who did or did not achieve remission over the 6 months follow-up period.



Figure 1. Six month DAS28CRP remission versus pre-treatment median fluorescence of CD39 expression on CD4+ T-cells (A); CD25 High expressing CD4+ T-cells (B); and ROC curve of predictive utility of pre-treatment CD39 expression on CD25 High CD4+ T-cells (C).

Conclusion: These findings support the potential role of CD39 in the mechanism of methotrexate response. Expression of CD39 on circulating Tregs in treatment-naïve RA patients may have particular value in identifying early RA patients likely to respond to methotrexate, and hence add value to evolving multi-param eter discriminatory algorithms.

\section{REFERENCES:}

[1] Hazlewood GS, et al. BMJ. 2016 21;353:11777

[2] Brown PM, et al. Nat Rev Rheumatol. 2016;12(12):731-742

[3] Peres RS, et al. Proc Natl Acad Sci U S A. 2015;112(8):2509-2514

Disclosure of Interests: None declared

DOI: 10.1136/annrheumdis-2021-eular.2030

\section{OP0034 STP938, A NOVEL, POTENT AND SELECTIVE INHIBITOR OF CTP SYNTHASE 1 (CTPS1) DEMONSTRATES EFFICACY IN RODENT MODELS OF INFLAMMATION AND ARTHRITIS}

H. Asnagli ${ }^{1}$, A. Novak ${ }^{2}$, L. Birch ${ }^{2}$, R. Lane ${ }^{2}$, N. Minet ${ }^{3}$, D. Laughton ${ }^{2}$, P. George ${ }^{4}$ G. De Ribains ${ }^{5}$, S. Latour ${ }^{3}$, A. Fischer ${ }^{6}$, T. Bourne ${ }^{7}$, A. Parker ${ }^{1}$. ${ }^{1}$ Step Pharma, Pharmacology, Archamps, France; ${ }^{2}$ Sygnature Discovery, Discovery BuildingBiocity Building, Nottingham, United Kingdom; ${ }^{3}$ Institut Imagine, Laboratory of Lymphocyte Activation \& EBV Susceptibility, INSERM-U1163, Paris, France; ${ }^{4}$ Independent consultant, Drug Discovery \& Development, Paris, France; ${ }^{5}$ Gamut, Therapeutics, Paris, France; ${ }^{6}$ INSERM-U1163, AP-HP, Paris, France; ${ }^{7}$ Milvuswood, Consultancy, High Wycombe, United Kingdom

Background: The final rate-limiting step in pyrimidine synthesis is the conversion of UTP to CTP which is catalyzed by cytidine triphosphate synthase 1 (CTPS1) or CTPS2. A hypomorphic mutation in the CTPS1 gene has highlighted the essential and non-redundant role of CTPS1 in T and B lymphocyte proliferation ${ }^{1}$. These patients exhibit no effects on non-hematopoietic tissues. Thus, selective inhibition of CTPS1 represents a novel targeted approach to dampen pathological T- and B-cell lympho-proliferation. STP938 is an orally bioavailable, small molecular weight, selective inhibitor of CTPS1 developed by Step Pharma. Objectives: To demonstrate the in vitro effects of CTPS1 inhibition on T and B cell proliferation and the therapeutic potential of STP938 using in vivo models of disease.

Methods: The in vitro anti-proliferative activity of STP938 was investigated using cell lines and primary human PBMCs. STP938 was assessed in vivo using the $\mathrm{DTH}-\mathrm{KLH}$ rat model and the mouse collagen-induced arthritis (CIA) model. For the KLH-DTH model, Lewis rats were immunized with $\mathrm{KLH}$, a week later, challenged locally at the ear with KLH antigen, ear swelling was assessed after 24 hours. Blood samples were collected for detection of KLH-specific IgG levels at day 8. STP938 was given orally one-hour prior to immunization and then b.i.d. for 7 days. For the CIA model, DBA-1 mice were immunized with Collagen type II and complete Freund's adjuvant and received a booster immunization three 